\title{
Intrapulse Dynamical Effects in Multiphoton Processes: Theoretical Analysis
}

\author{
Jianshu Cao,* Jianwei Che, and Kent R. Wilson \\ Department of Chemistry and Biochemistry, University of California, San Diego, \\ LaJolla, California 92093-0339
}

Received: September 22, 1997

\begin{abstract}
Stationary and nonstationary multilevel models are formulated to analyze ultrafast multiphoton processes with emphasis on electronic and nuclear coherence of a near-resonant intermediate state. By identifying and characterizing three intrapulse dynamical mechanisms (sequential-resonance, chirp-following, and time-delay), a clear physical picture is developed for understanding the subtle chirp dependence in intrapulse pumpprobe processes. The theoretical analysis in the paper provides not only a possible interpretation for a recent three-photon experiment but also a general theoretical framework for studying ultrafast multiphoton processes in atoms and molecules.
\end{abstract}

\section{Introduction}

Femtosecond chemistry offers experimentalists the opportunity to study elementary chemical processes on the molecular level and to directly monitor the dynamical evolution from reactants to products. ${ }^{1,2}$ Femtosecond excitation creates classicallike molecular wave packets, which are the basis of understanding the dynamics of chemical reactions: how they take place and how to govern them. ${ }^{3-6}$ Laser pulses can be focused into extremely narrow spatial and temporal distributions, and thus multiphoton excitation is common and is an important subject for study. In this paper, we present theoretical models and semiclassical analyses of dynamical effects of excited molecular wave packets in multiphoton processes, with special emphasis on those related to chirp, the variation of frequency with time.

Several theoretical and experimental studies have revealed that frequency-modulated laser pulses can introduce remarkable dynamic effects in molecular systems. Even in the weak response limit, the sign of the chirp proves crucial in wave packet focusing and defocusing, even though the total population being excited is a function only of the power spectrum. ${ }^{7}$ In a pumpdump or pump-probe experiment, the chirp of the second pulse can be optimized to follow the motion of the wave packet induced by the pump pulse, hence improving the quantum yield substantially. ${ }^{8,9}$ This chirp following effect becomes an intrinsic feature of multiphoton and strong-field optical processes. It has been demonstrated both numerically and experimentally that negatively chirped pulses induce large-amplitude vibrational motion on the ground electronic state through an effective intrapulse pump-dump mechanism. ${ }^{10,11}$ In light of this mechanism, we have shown that nearly complete electronic population transfer in molecular systems can be achieved with positivelychirped broadband intense laser pulses. ${ }^{12}$ Surprisingly, such molecular $\pi$ pulses are extremely robust and stable under thermal and dissipative conditions. In addition to chirp following within a single pulse, we will demonstrate in this paper that multiphoton processes exhibit unique features of electronic and nuclear coherence such as intrapulse sequential resonance and intrapulse time delay. In a recent three-photon experiment, intriguing results have been observed which can be understood only within a dynamical picture of laser-induced coherence. ${ }^{13}$ In short, chirp-dependent effects in multiphoton processes provide us a means to explore the coherence between laser fields and molecular systems.

To study dynamical effects in ultrafast multiphoton processes, a simple model consisting of a three-electronic state molecular system interacting with a Gaussian electric field is introduced in section A. The primary feature of femtosecond spectroscopy is the extremely short time duration of laser pulses such that the nuclear wave packet can often be assumed frozen during excitation. ${ }^{14-16}$ Under this assumption, the three-electronic state model is reduced to a stationary three-level problem, just as the two-level model is used for linear absorption. This classical model is then applied to two-photon absorption in section B, where we show a chirp-dependent enhancement due to the sequential-resonance with the intermediate state. As investigated in a previous paper, ${ }^{9}$ quantum dynamical corrections to the frozen wave packet approximation are necessary for linear absorption from a nonstationary initial state, as in the probe process of pump-probe experiments. In section $\mathrm{C}$, a nonstationary single-photon theory is formulated to illustrate the general principle of chirp following. In single-pulse multiphoton processes, since the same electric field creates an accelerating wave packet and then excites it further onto the final state, it is necessary to treat both stages of excitation consistently within the semiclassical framework. To do this, a two-photon nonstationary approximation is derived in section $\mathrm{D}$ with emphasis on the induced motion on the intermediate electric surface. Application of the nonstationary expression to the intrapulse pump-dump process in section E reveals the effect of intrapulse chirp following. By virtue of the nonstationary model, a comprehensive theory of two-photon absorption is derived in section $\mathrm{F}$, where the chirp-dependence is shown to be the result of all three intrapulse coherent mechanisms (sequentialresonance, chirp-following, and time-delay). A final analysis in section $\mathrm{G}$ of a three-photon process reveals essentially the same physics as the intrapulse pump-probe process, which provides a possible explanation for the experimental observations of a three-photon absorption experiment by Yakovlev, Bardeen, Che, Cao, and Wilson. ${ }^{13}$

\section{Analysis}

A. Stationary Three-Level Model. As a generic model, consider a molecule with three electronic states described by 
Two-photon Absorption

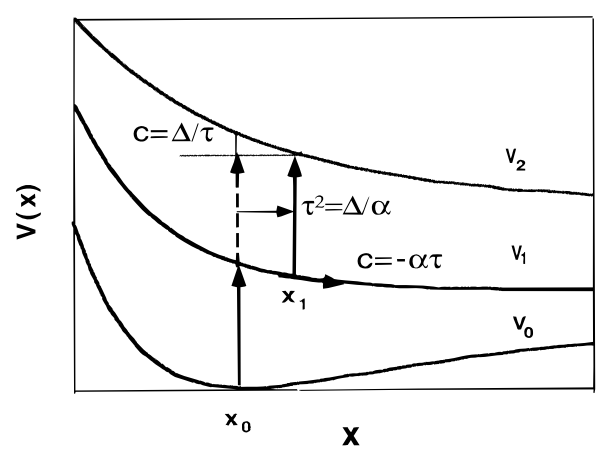

Figure 1. A schematic diagram of a two-photon process where a molecule in the ground state $|0\rangle$ is excited by a nearly resonant electric field to a final electronic state $|2\rangle$ via an intermediate state 1$\rangle. x_{0}$ is the center of the Franck-Condon regime, or equivalently the center of the ground-state distribution, and $x_{1}$ is the displaced center of the excited wave packet. The three intrapulse dynamical mechanisms (from top to bottom, sequential-resonance, time-delay, and chirp following) are illustrated with corresponding expressions.

three electronic Hamiltonians, $\hat{H}_{0}$ for the ground state $|0\rangle, \hat{H}_{0}$ for the intermediate excited state $|1\rangle$, and $\hat{H}_{2}$ for the final state $|2\rangle$. As shown schematically in Figure 1 and summarized in section $\mathrm{F}$, this three-level molecule then couples via a dipole interaction to a time-dependent electric field which is treated classically as $\epsilon(t)=E(t)+E^{*}(t)$. By definition, two-photon absorption is the excitation process from the ground state $|0\rangle$ through the intermediate state $|1\rangle$ to the final state $|2\rangle$. Because the fluorescence detection time $t_{f}$ is set to be much larger than the pulse duration $\tau$, the wave function at $t_{f}$ can be expressed as $\psi_{2}\left(t_{f}\right)=\mathrm{e}^{-\mathrm{i} \hat{H}_{2} t_{\mathrm{f}} / \hbar} \psi_{2}$, where $\psi_{2}$ is given, to second order in the dipole interaction, as

$$
\psi^{(2)}=(i / \hbar)^{2} \int_{-\infty}^{\infty} \mathrm{d} t_{2} \int_{-\infty}^{t_{2}} \mathrm{~d} t_{1} \hat{G}\left(t_{2}, t_{1}\right) \epsilon\left(t_{2}\right) \epsilon\left(t_{1}\right) \psi_{0}
$$

Here, $\hat{G}$ is a two-time Green function defined as

$$
\hat{G}\left(t_{2}, t_{1}\right)=\mathrm{e}^{\mathrm{i} \hat{H}_{2} t_{2} / \hbar} \mathrm{e}^{-\mathrm{i} \hat{H}_{1}\left(t_{2}-t_{1}\right) / \hbar} \mathrm{e}^{-\mathrm{i} \hat{H}_{0} t_{1} / \hbar}
$$

where the transition dipole moments are assumed to be constants and are omitted without causing any confusion. The electric field in eq 1 is assumed to take the general form of a chirped Gaussian pulse, defined as

$$
E(t)=E_{0} \exp \left[-\frac{t^{2}}{2 \tau^{2}}-\mathrm{i} \omega_{0} t-\mathrm{i} c \frac{t^{2}}{2}\right]
$$

where four parameters are employed to characterize the light pulse: an amplitude $E_{0}$, a carrier frequency $\omega_{0}$, a temporal width $\tau$, and a linear chirp rate $c$, respectively. The chirp describes the drift of the instantaneous frequency with time and thus represents phase modulation in the laser field. The Fourier transform of the field gives

$$
\begin{aligned}
\tilde{E}(\omega)=\tilde{E}_{0} \exp [- & \left.\frac{\left.\left(\omega-\omega_{0}\right)^{2}\right]}{2 \gamma}\right]= \\
& \tilde{E}_{0} \exp \left[-\frac{\left(\omega-\omega_{0}\right)^{2}}{2 \Gamma}-\mathrm{i} c^{\prime} \frac{\left(\omega-\omega_{0}\right)^{2}}{2}\right]
\end{aligned}
$$

where $\gamma$ is the complex frequency width defined as $\gamma^{2}=1 / \tau^{2}$ $+\mathrm{i} c$ with $\operatorname{Re}\left(1 / \gamma^{2}\right)=1 / \Gamma^{2}$ and $\operatorname{Im}\left(1 / \gamma^{2}\right)=c^{\prime}$. By definition, the bandwidth is given as $\Gamma^{2}=1 / \tau^{2}+c^{2} \tau^{2}$ and the linear frequency chirp rate is given as $c^{\prime} \Gamma^{2}=c \tau^{2}$. For simplicity, the analysis in this section is formulated in the time domain representation. However, under real experimental conditions, the electric field is often characterized by its frequency chirp $c^{\prime}$ based on a fixed power spectrum with a given bandwidth $\Gamma$. The Fourier transformation between eqs 3 and 4 allows us to establish relations between temporal and frequency domain parameters. For example, the pulse duration $\tau$ is given by $\tau^{2}$ $=1 / \Gamma^{2}+\left(c^{\prime} \Gamma\right)^{2}$, indicating that $\tau$ increases with the linear frequency chirp rate $c^{\prime}$. By the same token, to conserve the integrated intensity $P=\pi E_{0}^{2} \tau$, the peak field amplitude $E_{0}$ decreases with $\tau$ or equivalently with $c^{\prime}$. The Gaussian field and related features discussed here will be useful for obtaining closed-form analytical results.

With the progress of ultrafast laser techniques, the pulse duration can be sufficiently short that the nuclear configuration is approximately frozen during the impulsive excitation. This observation constitutes the core assumption underlying the frozen wave packet approximation of linear absorption, namely, the stationary two-level-system models. ${ }^{14,15,9}$ By virtue of the same approximation, we ignore the kinetic energy operators in the two-time Green function and recast eq 2 as

$$
\hat{G}\left(t_{2}, t_{1}\right)=\exp \left[\mathrm{i} \omega_{2}(x) t_{2}+\mathrm{i} \omega_{1}(x) t_{1}\right]
$$

where $\hbar \omega_{1}(x)=V_{1}(x)-V_{0}(x)$ are $\hbar \omega_{2}(x)=V_{2}(x)-V_{1}(x)$ are the vertical transition energies between the two electronic states involved, respectively. In evaluating the population on the final state, the relevant quantity is the average of the Green functions over the distribution $\rho_{0}(x)$ on the ground-state surface. The initial distribution is usually well-localized around the center of the Franck-Condon regime at $x_{0}$ so that the average over $\rho_{0}(x)$ can be replaced by the value evaluated at $x_{0}$, giving

$$
\operatorname{Tr}\left[\hat{G}^{+}\left(t_{2}^{\prime}, t_{1}^{\prime}\right) \hat{G}\left(t_{2}, t_{1}\right) \rho_{0}\right] \approx G_{0}^{+}\left(t_{2}^{\prime}, t_{1}^{\prime}\right) G_{0}\left(t_{2}, t_{1}\right)
$$

where the plus sign denotes the corresponding complex conjugate. Here, the value of $G_{0}$ at $x_{0}$ is explicitly given as

$$
\hat{G}\left(t_{2}, t_{1}\right) \rightarrow G_{0}\left(t_{2}, t_{1}\right)=\exp \left[\mathrm{i} \omega_{2}\left(x_{0}\right) t_{2}+\mathrm{i} \omega_{1}\left(x_{0}\right)\right]
$$

where the equivalence indicated by the arrow holds only for the evaluation of the final population. The simple three-level expression in eq 7 is the starting point for the two-photon absorption discussed in section B.

B. Stationary Analysis of Two-Photon Absorption: Intrapulse Sequential Resonance. The two-photon analysis presented here serves as a basis for understanding other nonlinear processes, including those described in later subsections. By virtue of the frozen wave packet approximation, we have shown in a previous paper ${ }^{9}$ that two-photon absorption can be simplified to a single-photon process with an effective field and with a prefactor which is only a function of the spectrum, if the off-resonant detuning is much larger than the bandwidth. Here, we review the three-level-model of twophoton absorption and demonstrate the correlation between the sign of the chirp and the off-resonant detuning of an intermediate level.

The relevant quantity for study in this paper is the integrated fluorescence yield or equivalently the population on the final electronic state. On substituting the three-level expression of eq 7 into the second-order wave function in eq 1, we immediately obtain the expression for the population on the final state

$$
N_{2}=\left|\int_{-\infty}^{\infty} \mathrm{d} t_{2} \int_{-\infty}^{t_{2}} \mathrm{~d} t_{1} \mathrm{e}^{\mathrm{i} \omega_{2} t_{2}+\mathrm{i} \omega_{1} t_{1}} E\left(t_{2}\right) E\left(t_{1}\right)\right|^{2}
$$


where $\omega_{1}$ and $\omega_{2}$ are the vertical frequencies of the first and second transitions at the center of the Franck-Condon region. With the introduction of a Gaussian pulse as in eq 3 and the change of integral variables $s=t_{2}-t_{1}$ and $2 t=t_{2}+t_{1}$, the double integrals of $t$ and $s$ in eq 8 can be separated, giving

$$
N_{2}=\left|C(\Delta) \int_{-\infty}^{\infty} \mathrm{d} t E^{2}(t) \mathrm{e}^{\mathrm{i} t\left(\omega_{2}+\omega_{1}\right)}\right|^{2}
$$

Here, $C(\Delta)$ is the result of the $s$ integration, explicitly given by

$$
C(\Delta)=\int_{0}^{\infty} \mathrm{d} s \exp \left[-\frac{s^{2}}{4}\left(\frac{1}{\tau^{2}}+\mathrm{i} c\right)+\mathrm{i} \frac{\Delta s}{2}\right]
$$

with $\Delta=\omega_{2}-\omega_{1}$ being the detuning of the intermediate state. When the detuning is much larger than the bandwidth (i.e., $\Delta$ $\gg \Gamma$ ) the absolute value of $C(\Delta)$ is a function of the bandwidth and detuning only. Though derived for Gaussian pulses, the conclusion is general: If the intermediate electronic state is faroff-resonant, any two-photon process can be viewed as an singlephoton process with an effective field $E^{2}(t)$ and with an effective transition dipole which includes the prefactor of $C(\Delta)$. Consequently, the spectrum of two-photon absorption can be expressed as

$$
Q(\omega)=\left|\int \tilde{E}\left(\omega^{\prime}\right) \tilde{E}\left(\omega-\omega^{\prime}\right) \mathrm{d} \omega^{\prime}\right|^{2}
$$

which, in contrast to linear absorption, is a function of the frequency chirp but independent of its sign. This chirp dependence in two-photon absorption has been predicted and observed experimentally. ${ }^{17}$

On completing the integral in eq 9 , we obtain $N_{2} \propto P^{2} / \tau \Gamma$ for $\Delta \gg \Gamma$, indicating an inverse dependence on the pulse duration for a fixed power spectrum. This agrees with the observation that the yield of two-photon absorption depends on the peak intensity, which decreases with the pulse duration. From this consideration, the transform-limited pulse with a minimal $\tau$ is preferable. However, when the intermediate state is on resonance, $\Delta=0$, the integration of $s$ in $|C(\Delta)|^{2}$ contributes a factor $|C(\Delta)|^{2} \propto \tau / \Gamma$ and we have $N_{2} \propto P^{2} / \Gamma^{2}$ which is independent of the frequency chirp. In general, for an $n$-photon process with $l$ resonances including the final state, the final population is given by $N \propto P^{n} / \Gamma^{l} \tau^{n-l}$, indicating resonance enhancement in intensity dependence.

An interesting situation arises when the detuning is comparable to the bandwidth, (i.e., $\Delta \approx \Gamma$ ). The prefactor $C(\Delta)$ in eq 10 can be expressed in terms of a complex error function and is generally a function of the chirp. The absolute value of $C(\Delta)$ is maximized when the imaginary part of the exponent in eq 10 approaches zero, that is, $\left(s \Delta / 2-c s^{2} / 4\right) \approx 0$. Using the pulse duration $\tau$ for the average value of the $s$ integral, (i.e., $s$ $\propto \tau$ ) we obtain an estimation for the optimal linear chirp rate

$$
c \propto \frac{\Delta}{\tau}
$$

indicating a correlation between the sign of the chirp and the off-resonance detuning of the intermediate state. This correlation can be understood by the fact that the quantum yield of multiphoton processes is enhanced dramatically by resonance with an intermediate state. Due to the optimal chirp in eq 12, the drift of the instantaneous frequency of the laser field is such that in the first half pulse it is resonant with the first transition and in the second half pulse with the second transition. As demonstrated in Figure 2, the matching of the phase modulation in the electric field and the detuning of the electronic levels
Intra-Pulse Transient Resonance
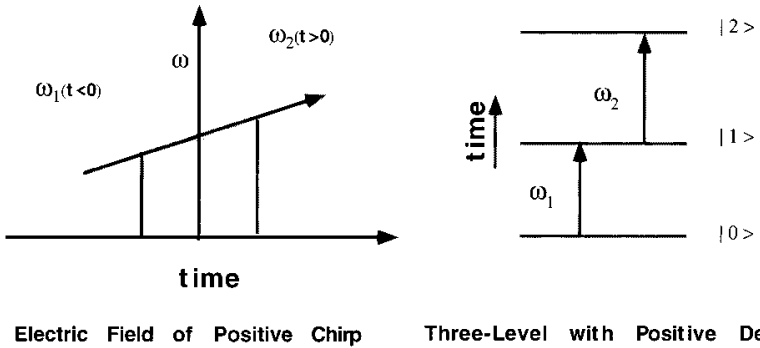

Figure 2. An illustration of intrapulse sequential resonance. The left image represents a positively chirped electric field $\left(c^{\prime}>0\right)$ and the right image represents a positive detuning of the three-electronic-state system $(\Delta>0)$. When chirp and the detuning are matched, a transient population can be built up on the intermediate electronic state so that the efficiency of two-photon excitation is enhanced dramatically.

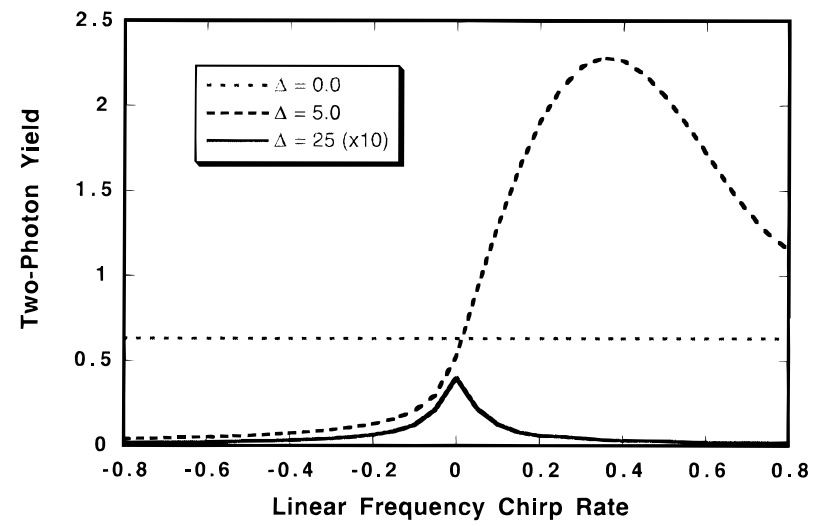

Figure 3. A plot of the two-photon yield $N_{2}$, evaluated from eqs 9 and 10 as a function of the linear frequency chirp rate $c^{\prime}$. The parameters are taken as $\Gamma=5$ and $P^{2} / \Gamma=1$, and the final state is assumed to be resonant. The three curves correspond to a resonant electric field $(\Delta=$ $0)$, a near-resonant electric field $(\Delta=5)$, and a far-off-resonant electric field $(\Delta=25)$, respectively. The result for $\Delta=25$ is shown after a magnification of 10 times.

results in a dynamical resonance which can build up transient population in the intermediate state though the carrier frequency is off-resonant. Unlike other intrapulse mechanisms we will discuss later, the intrapulse sequential resonance arises from electronic coherence rather than nuclear coherence and therefore can be fully described within the stationary three-level model.

As an illustrative example, the two-photon yield $N_{2}$ evaluated from eqs 9 and 10 is plotted in Figure 3 as a function of the linear frequency chirp rate $c^{\prime}$. The parameters are taken as $\Gamma$ $=5$ and $P^{2} / \Gamma=1$ and the final state is assumed to be on resonance. As expected, for a resonant intermediate electronic state $(\Delta=0)$, the yield is a constant independent of $c^{\prime}$. With a moderate detuning $(\Delta=5)$, the peak at a positive chirp rate confirms the relationship in eq 12 resulting from intrapulse sequential resonance. For the case of large detuning $(\Delta=25)$, the yield is significantly reduced and exhibits an inverse dependence on the pulse duration.

In most two-photon absorption processes, the detuning is sufficiently large that the sign effect of the chirp is insignificant. However, if the intermediate state becomes resonant or nearly resonant, the vibrational coherence of the molecular wave packet created by the first photon also become important. Therefore, the chirp dependence in two-photon absorption is the result of the two competing mechanisms, which will be the topic of section F. 
C. Linear Theory of Chirp Following. In contrast to linear-response excitation from a stationary ground electronic state, the excited state population resulting from linear excitation of a nonstationary wave packet correlates strongly to the chirp. Since the nonstationary wave packet has to be prepared by a pump pulse, the full treatment of pump-probe experiments should be carried out within the framework of nonlinear absorption. Nevertheless, if the pulse durations of pump and probe fields are much shorter than the delay time between the two pulses, the sequential pump and probe processes can be treated as two separated linear optical processes. With this consideration, in a previous paper, ${ }^{9}$ we have incorporated higher order quantum correction terms into the classical two-levelsystem model and thus generalized the frozen wave packet approximation to treat nonstationary wave packets. On the basis of this approach, we have theoretically justified the relation between the linear chirp rate of the optimal probe pulse and the velocity of the wave packet being probed, which has been predicted earlier by numerical simulation. ${ }^{8}$ This chirp following effect proves to be an important aspect of nuclear coherence in the nonlinear optical processes discussed in this paper. For this reason, we present here a nonstationary linear absorption model to supplement the analysis in the previous paper. ${ }^{9}$

For a wave packet whose center moves on a given trajectory $x(t)$, the population excited by a probe field $E_{2}(t)$ can be written as

$$
\begin{array}{r}
N_{2}=\int \mathrm{d} t_{2}^{\prime} \int \mathrm{d} t_{2}\left\langle\psi_{1}\left(t_{2}^{\prime}\right) \mid \mathrm{e}^{-\mathrm{i} \hat{H}_{2}\left(t_{2}^{\prime}-t_{2}\right) / \hbar} \psi_{1}\left(t_{2}\right)\right\rangle E^{*}\left(t_{2}^{\prime}\right) E\left(t_{2}\right) \\
=\int \mathrm{d} t \int \mathrm{d} s\left\langle\psi_{1}(t)\left|\mathrm{e}^{\mathrm{i} \hat{H}_{2} s / 2} \mathrm{e}^{-\mathrm{i} \hat{H}_{1} s / \hbar} \mathrm{e}^{\mathrm{i} \hat{H}_{2} s / 2}\right| \psi_{1}(t)\right\rangle E^{*}(t+ \\
s / 2) E(t-s / 2)
\end{array}
$$

where $s=t_{2}^{\prime}-t_{2}$ and $2 t=t_{2}^{\prime}+t_{2}$. The operator in the bracket is symmetric and therefore can be expanded to second order as $\mathrm{e}^{\mathrm{i} s \omega_{2}(x)}$ with $\hbar \omega_{2}(x)=H_{2}-H_{1}$. Assuming that the wave packet is well-localized so that the average over the probability distribution can be replaced by the expression evaluated at the center of the distribution, we have

$$
\begin{array}{r}
N_{2} \approx \int \mathrm{d} t \int \mathrm{d} s \mathrm{e}^{-\mathrm{i} s \omega[x(t)]} E^{*}(t+s / 2) E(t-s / 2)= \\
\int \mathrm{d} t F\left\{t, \omega_{2}[x(t)]\right\}
\end{array}
$$

where $F(t, \omega)$ is known as the Wigner transform of the probe field. To proceed, we use a general Gaussian form for the probe field centered at time $t_{0}$,

$$
E(t)=E_{0} \exp \left\{-\frac{\left(t-t_{0}\right)^{2}}{2 \tau^{2}}-\mathrm{i} \int_{t_{0}}^{t} \omega\left(t^{\prime}\right) \mathrm{d} t^{\prime}\right\}
$$

whose Wigner transform is given as

$$
F(t, \omega)=E_{0}^{2} \exp \left[-\frac{\left(t-t_{0}\right)^{2}}{\tau^{2}}-[\omega-\omega(t)]^{2} \tau^{2}\right]
$$

Substituting the above expression into eq 14, we find the optimal condition for the maximum population

$$
\omega_{2}[x(t)]=\omega(t)
$$

which indicates that the instantaneous frequency of the pulse should follow the vertical transition frequency at the center of the moving wave packet. As a general expression for chirp following, eq 17 implies a match of the wave packet motion to the frequency drift at all times, or equivalently to all orders of the chirp. For example, ${ }^{8,9}$ expanding eq 17 at $t_{0}$ to zero and linear orders, we have $\omega_{2}\left(x_{0}\right)=\omega\left(t_{0}\right)$ and $v\left(x_{0}\right) \mathrm{d} \omega_{2}\left(x_{0}\right) / \mathrm{d} x=$ $c\left(t_{0}\right)$, with $x_{0}=x\left(t_{0}\right)$ and $v\left(x_{0}\right)=\mathrm{d} x\left(t_{0}\right) / \mathrm{d} t$. In fact, by optimizing the chirp at a given time $t_{0}$, one can estimate the local potential energy surface and, by repeating the procedure at various delay times, one can in principle map out the potential energy surface along the trajectory $x(t)$.

D. Nonstationary Three-Level Model. As long as the pulse duration is sufficiently short, the frozen wave packet approximation is useful for describing linear and nonlinear absorption if only electronic coherence is concerned. However, to reflect the nuclear coherence in multiphoton processes, one must take into account the crucial difference that the wave packet on an excited state potential surface is in motion whereas the original wave packet on the ground state potential surface is at rest. This consideration leads to a nonstationary analysis as follows.

The basic quantum propagator for any linear or nonlinear optical excitation can be written as

$$
\hat{K}(t)=\mathrm{e}^{\mathrm{i} t \hat{H}^{\prime} / \hbar} \mathrm{e}^{-\mathrm{i} t \hat{H} / \hbar}
$$

where, as before, the transition dipole operator is assumed as a constant. Any optical process can be formulated as the action of this propagator or its product on the initial wave function integrated over time variables with the corresponding laser fields. Making use of the well-known operator identity $\mathrm{e}^{\hat{A}} \mathrm{e}^{\hat{B}}=$ $\mathrm{e}^{\hat{A}+\hat{B}+1 / 2[\hat{A}, \hat{B}]}$, we have

$$
\hat{K}(t)=\mathrm{e}^{\mathrm{i} V(x) t / 2 \hbar} \mathrm{e}^{-\mathrm{i} \hat{\eta}(x) \hat{p} / \hbar} \mathrm{e}^{\mathrm{i} V(x) t / 2 \hbar}
$$

where $V=H^{\prime}-H$ is the potential difference, $\hat{p}$ is the momentum operator, and $\eta(x)=-t \nabla V(x) / m$, with $\nabla$ being the spatial derivative, is the displacement of the wave packet due to the acceleration on the excited electronic surface during the excitation. When applied to the wave function $\psi(x)$, eq 19 leads to

$$
\hat{K}(t) \psi(x)=\exp \left\{\frac{\mathrm{i} t}{2 \hbar}\left[V(x)+V\left(x^{\prime}\right)\right]\right\} \psi\left(x^{\prime}\right)
$$

where $x^{\prime}=x-\eta(t)$ includes a semiclassical correction because of the noncommutation of the kinetic and potential energy operators. If the second-order quantum correction is ignored, the wave packet is stationary during excitation and thus the stationary three-level expression in section A can be recovered. It is therefore not surprising that the nonstationary expression in eq 20 , when applied to detecting wave packet motion in pump-probe experiments, leads to exactly the same result as the generalized Bersohn-Zewail model proposed in a previous paper. ${ }^{9}$

It is well-known that the total linear absorption cross section, or equivalently, the total fluorescence yield of single-photon excitation, is only a function of the power spectrum of the laser field and hence independent of its frequency chirp. ${ }^{18}$ However, as indicated by eq 20 , the chirp has significant effects on the phase space distribution of the excited state wave function (i.e., the position and momentum of the wave packet created on the excited state surface). From the viewpoint of the eigenstate distribution, the chirp modulates the phase coherence among the eigenlevels of the excited wave function, which cannot be detected from the total LIF yield of single-photon absorption but can be measured by second and higher order optical processes.

The operator expression in eq 20 is general and applies to all linear and nonlinear optical processes. For the special case 
of two-photon optical excitation involving three electronic states, further approximations lead to a simpler but equally effective expression. To be specific, we notice that the initial wave packet is stationary on the ground electronic surface and that the wave packet motion on the final electronic surface is irrelevant as far as the final population is concerned. Consequently, the kinetic energy operators in the initial and final Hamiltonians can be ignored and only the wave packet motion on the intermediate electronic surface needs to be considered. By expanding the quantum operator on the intermediate state to second order in time, we can reduce the two-time Green function in eq 2 to

$$
\hat{G}\left(t_{2}, t_{1}\right)=\mathrm{e}^{\mathrm{i} \omega_{2}(x) t_{2}} \exp \left[-\mathrm{i} \hat{\eta}(x) \hat{p} / \hbar+O\left(s^{3}\right)\right] \mathrm{e}^{\mathrm{i} \omega_{1}(x) t_{1}}
$$

where $\hbar \omega_{1}(x)=V_{1}(x)-V_{0}(x), \hbar \omega_{2}(x)=V_{2}(x)-V_{1}(x), s=t_{2}$ $-t_{1} \geq 0$, and $\eta(x)=f_{1}(x) s^{2} / 2 m$ which is the displacement of the wave packet due to the force $f_{1}(x)=-\nabla V_{1}(x)$ on the excited state $|1\rangle$ during the excitation. In evaluating the population on the final state $|2\rangle$, a similar argument as in eq 7 leads to

$$
\hat{G}\left(t_{2}, t_{1}\right) \rightarrow G_{0}\left(t_{2}, t_{1}\right)=\exp \left[\mathrm{i} \omega_{2}\left(x_{1}\right) t_{2}+\mathrm{i} \omega_{1}\left(x_{0}\right)\right]
$$

with $x_{1}=x_{0}+\eta\left(x_{0}\right)$ being the displaced center of the wave packet on the excited state surface. On expanding $\omega_{2}\left(x_{1}\right)$ to linear order, we obtain

$$
\omega_{2}\left(x_{1}\right) \approx \omega_{2}\left(x_{0}\right)-\alpha\left(x_{0}\right) s^{2}
$$

with $\alpha\left(x_{0}\right)=\nabla \omega_{2} \nabla V_{1} / 2 m$. In comparison with the expression in eq 7 , the second term in eq 22 represents a quantum correction to the classical three-level model, that is, the nonstationary effect. Equation 2 along with eq 23 forms one of the central results of this work which is used throughout the rest of the paper.

E. Beyond Linear Absorption: Intrapulse Chirp Following. As the intensity of a light field increases, the optical excitation process starts to deviate from the linear response regime. The leading order correction to linear absorption is population back-transfer from the excited to the ground electronic state [i.e., the intrapulse pump-dump mechanism discussed by Ruhman and Kosloff, ${ }^{10}$ which is the simplest case of a second-order process). We include it here to exemplify the general principle of chirp following within a single electric field.

The second order expression for the population on the ground state can be written, under the rotating wave approximation, as

$$
N_{0}^{(2)}=\left|\int_{-\infty}^{\infty} \mathrm{d} t_{2} \int_{-\infty}^{\infty} \mathrm{d} t_{1} \hat{G}\left(t_{2}, t_{1}\right) E^{*}\left(t_{2}\right) E\left(t_{1}\right)\right|^{2}
$$

which is similar to eq 8 except that the final state is the same as the initial ground state, $|2\rangle$. Following the same argument in eqs 22 and 23, the Green function in eq 24 becomes

$$
\hat{G}\left(t_{2}, t_{1}\right) \rightarrow G_{0}\left(t_{2}, t_{1}\right) \approx \exp \left[-\mathrm{i} \omega_{1}\left(x_{0}\right) t_{2}+\mathrm{i} \alpha s^{2} t_{2}+\mathrm{i} \omega_{1}\left(x_{0}\right) t_{1}\right]
$$

where identity $\omega_{2}(x)=-\omega_{1}(x)$ is used and $s=t_{2}-t_{1}$. Since $x_{0}$ is the equilibrium center of the ground state distribution, we have $\nabla V_{0}\left(x_{0}\right)=0$ and hence

$$
\alpha=\nabla \omega_{1} \nabla V_{1} / 2 m=\left(\nabla V_{1}\right)^{2} / 2 m \hbar \geq 0
$$

where $x_{0}$ is omitted without causing confusion. Next, making use of the Gaussian functional form of the laser field in eq 3 and the linear transformation of $s=t_{2}-t_{1}$ and $2 t=t_{2}+t_{1}$, we obtain the expression for the back-transfer population
Intra-Pulse Pump-Dump

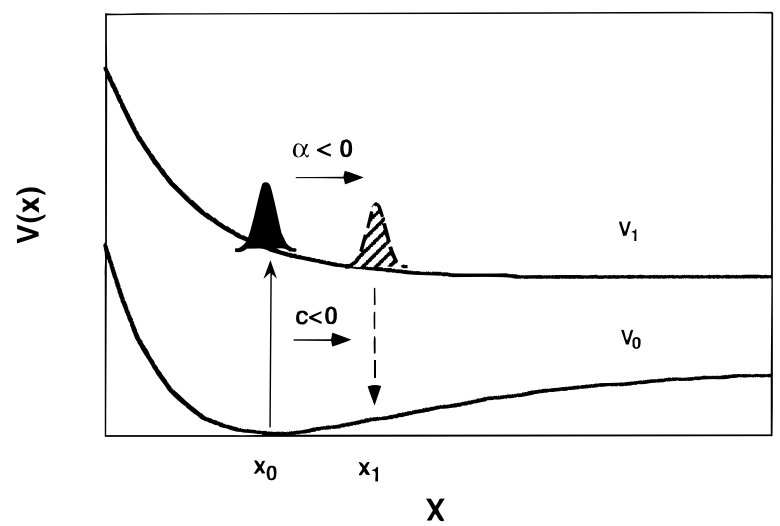

Figure 4. A schematic illustration of intrapulse pump-dump. With a displacement of the center of the excited wave packet from $x_{0}$ to $x_{1}$, the resonance frequency decreases accordingly $(\alpha<0)$. When this frequency shift is matched, a negatively chirped pulse leads to optimal back-transfer efficiency.

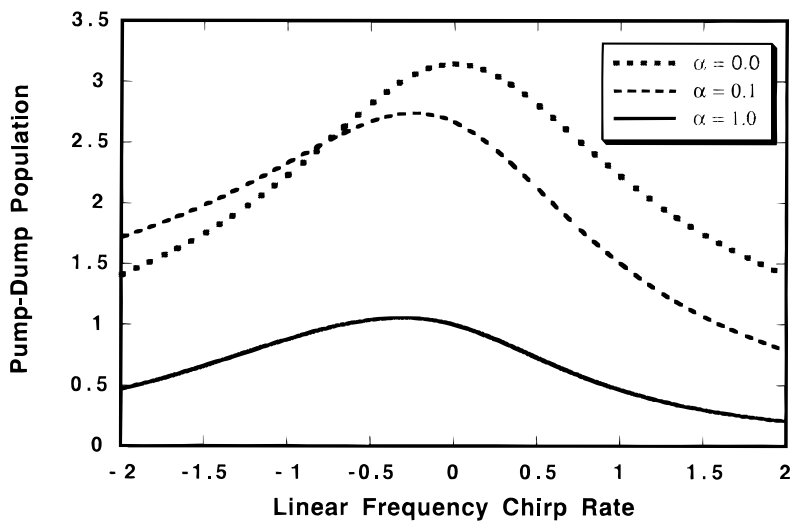

Figure 5. A plot of the pump-dump population in eq 27 as a function of the linear frequency chirp rate $c^{\prime}$ for a resonant electric field of $\Gamma=$ $1, \Delta=0$, and $P=1$. The three curves correspond to a stationary wave packet $(\alpha=0)$, a slow acceleration $(\alpha=0.1)$, and a fast acceleration $(\alpha=1)$, respectively.

$$
N_{0}^{(2)}=\frac{P^{2}}{\Gamma \tau}|C(\Delta)|^{2}
$$

where the integration over the $s$ variable is explicitly given as

$$
C(\Delta)=\int_{0}^{\infty} \mathrm{d} s \exp \left[-\frac{s^{2}}{4 \tau^{2}}-\frac{\tau^{2} s^{2}}{4}(c+\alpha s)^{2}-\mathrm{i} s \Delta+\mathrm{i} \frac{\alpha}{2} s^{3}\right]
$$

with the detuning $\Delta=\omega_{1}\left(x_{0}\right)-\omega_{0}$. Optimizing $N_{0}^{(2)}$ requires the minimization of the square term of the exponent in $C(\Delta)$ (i.e., $\left.(c+\alpha s)^{2} \approx 0\right)$. Since the average value of $s$ is on the order of the pulse duration $\tau$ (i.e., $s \propto \tau$ ) we then obtain an estimation for the optimal linear chirp rate as

$$
c \propto-\alpha \tau
$$

Because $\alpha$ given in eq 26 is positive, the optimal chirp is always negative.

The physical implication of eq 29 corresponds exactly to the optimal intrapulse pump-dump mechanism, as illustrated in Figure 4, where back transfer is optimized with the negative chirp which follows the wave packet's moving down the potential slope. As an example, the pump-dump population in eq 27 is plotted in Figure 5 as a function of the linear frequency chirp rate for a resonant electric field of $\Gamma=1, \Delta=$ 
0 , and $P=1$. Without nuclear motion, the stationary model with $(\alpha=0)$ results in a yield function of $N_{0}^{(2)} \propto 1 / \tau$, independent of the sign of the chirp. Once wave packet motion is incorporated, the peak of the curve shifts from zero chirp to the negative side, indicating that the optimal pulse has a negative chirp as the result of intrapulse chirp following.

As the intensity further increases, higher order corrections beyond second order become more important and nonperturbative effects such as Rabi oscillation and adiabatic inversion become dominant. In fact, the opposite argument to the pumpdump mechanism leads to a proposal of using positively chirped pulses for electron population inversion, which proves to be a key component in a newly developed $\pi$ pulse theory for molecular systems. ${ }^{12}$ Though derived in the framework of second-order theory, the relation in eq 29 remains valid in the strong response regime if $s$ is estimated using the time when the Rabi oscillation reaches the first peak.

F. Nonstationary Analysis of Two-Photon Absorption: Intrapulse Pump-Probe Time Delay and General Solution. Consider a special situation of two-photon absorption where the transition frequencies from the ground to the intermediate excited state and from the excited to final state are nearly the same and the laser frequency is resonant or close to resonant with both frequencies. In a sense, this process can be viewed as a coherent pump-probe process within a single electric field, in contrast with sequential pump-dump experiments using two delayed laser pulses. As indicated in section B, a detailed analysis is needed for a full account of subtle dynamical effects.

We begin by writing the population on the final state as

$$
N_{2}=\left|\int_{-\infty}^{\infty} \mathrm{d} t_{2} \int_{-\infty}^{t_{2}} \mathrm{~d} t_{1} \mathrm{e}^{\mathrm{i} \omega_{2}\left(x_{1}\right) t_{2}+\mathrm{i} \omega_{1}\left(x_{0}\right) t_{1}} E\left(t_{2}\right) E\left(t_{1}\right)\right|^{2}
$$

where the nonstationary expression in eq 22 is used. In eq 30 , $\omega_{1}\left(x_{0}\right)$ is the vertical frequency of the first transition at the center of the Franck-Condon region and $\omega_{2}\left(x_{1}\right)$ is the vertical frequency of the second transition at the center of the linearly accelerating wave packet created by the first transition. We assume that the carrier frequency of the field is in resonance with the first transition (i.e., $\left.\omega_{1}\left(x_{0}\right)=\omega_{0}\right)$ but is off-resonant with the second transition with the detuning given as $\Delta=$ $\omega_{2}\left(x_{0}\right)-\omega_{0}$. Again, making use of the linear expansion for $\omega_{2}$ in eq 23, substituting the Gaussian pulse in eq 30 and changing integration variables to $s=t_{2}-t_{1}$ and $2 t=t_{2}+t_{1}$, we can rearrange eq 30 in terms of $s$ and $t$. However, due to the $\eta$ term, the integrations of $s$ and $t$ are coupled to high orders and therefore cannot be separated, implying that the effective field as defined in eq 9 is no longer possible. On completing the integration of $t$, we obtain

$$
N_{2}=\frac{P^{2}}{\Gamma \tau}|C(\Delta)|^{2}
$$

where the $s$ integral is explicitly expressed as

$$
C(\Delta)=\int_{0}^{\infty} \mathrm{d} s \exp \left[-\gamma^{2} \frac{s^{2}}{4}-\frac{\left(\Delta-\alpha s^{2}\right)^{2}}{4 \gamma^{2}}+\mathrm{i}\left(\Delta-\alpha s^{2}\right) \frac{s}{2}\right]
$$

with $\gamma^{2}=1 / \tau^{2}+\mathrm{i} c$. This general solution in eqs 31 and 32 is the final result of this paper. Based on this model, maximizing the intrapulse pump-probe yield requires the optimization of pulse parameters such that the expression in eq 31 reaches a global maximum for a given set of conditions. The optimization of eq 31 is discussed as follows.
1. As stated earlier, for two-photon absorption with a nearresonant intermediate state, the quantum yield is independent of the frequency chirp or the pulse duration for a fixed power spectrum. This predication can be confirmed by integration of the first term of the exponent in eq 32. The contribution from the real part of the second term of the exponent in eq $32,-(\Delta$ $\left.-\alpha s^{2}\right)^{2} / 4 \Gamma^{2}$, with $\Gamma=1 / \tau^{2}+c^{2} \tau^{2}$, can be optimized by setting $\Delta-\alpha s^{2} \approx 0$. Using $s \propto \tau$, we obtain the estimate for the optimal pulse duration,

$$
\tau^{2}=\frac{\Delta}{\alpha}
$$

which is valid if $\Delta / \alpha>0$. Physically, such an optimal duration exists only if the excited wave packet moves in a direction which decreases the detuning. In a sense, this effect is the analogue of time delay in pump-probe experiments and is thus called the intrapulse time-delay mechanism. For a given power spectrum with a fixed $\Gamma$ and $P$, the pulse duration $\tau$ increases with the linear frequency chirp rate, as described by $\tau^{2}=1 / \Gamma^{2}$ $+c^{\prime 2} \Gamma^{2}$. Therefore, the yield, as a function of the linear frequency chirp rate, has a single peak for $\Delta / \alpha \leq 0$ and two peaks with opposite sign for $\Delta / \alpha \gg 0$. The intrapulse timedelay mechanism has been first observed and explained by Yakovlev, Bardeen, Che, Cao, and Wilson in a recent threephoton experiment. ${ }^{13}$

2. Since the pulse duration is a function of $c^{\prime 2}$, the analysis, based on the real part of the exponent in eq 32, cannot determine the sign of the optimal chirp when there are two peaks of opposite sign. The contribution from the imaginary part of the exponent in eq 32 can by optimized by setting $-c s^{2} / 4+(\Delta-$ $\left.\alpha s^{2}\right) s / 2 \approx 0$. Again, using the pulse duration $\tau$ for the value of $s$ (i.e., $s \propto \tau$ ) we find the expression for the optimal chirp

$$
c \propto \frac{\Delta}{\tau}-\alpha \tau
$$

which also gives the sign of $c^{\prime}$. It is easy to recognize that the first term in eq 34 is the same as eq 12 in section B, which results from the correlation between the detuning and the chirp (intrapulse sequential resonance), and the second term in eq 34 is the same as eq 29 in section E, which describes the correlation between the wave packet motion and the chirp (intrapulse chirp following). Therefore, the sign of the optimal chirp is the consequence of the two competing mechanisms. In the case of a single peak, $\Delta / \alpha \leq 0$, the contributions from the two mechanisms have the same sign and hence the sign of the optimal chirp remains the same. In the case of two peaks, $\Delta / \alpha$ $>0$, the contributions from the two mechanisms have the opposite sign so that the optimal chirp for the maximum yield changes sign as the detuning $\Delta$ increases.

The three dynamical effects from eq 32 (i.e., sequentialresonance, chirp-following, and time-delay) are illustrated schematically in Figure 1 and the overall chirp dependence in a pump-probe process is their combined result. To verify the analysis, we numerically evaluate the two-photon yield in eqs 31 and 32 as a function of the linear frequency chirp rate $c^{\prime}$ for $\Gamma=5, \alpha=1$, and $P^{2} / \gamma=1$. As shown in Figure 6, the optimal pulse for a resonant pulse $(\Delta=0)$ has a negative chirp as the result of intrapulse chirp following. With a small detuning $(\Delta$ $=2$ ), there are two peaks due to intrapulse time delay, with the optimal pulse being a negative chirp. As the detuning increases to $\Delta=4$, the intrapulse sequential resonance effect starts to dominate and the higher peak of the two is located on the positively chirp axis. The higher optimal peak remains positive 


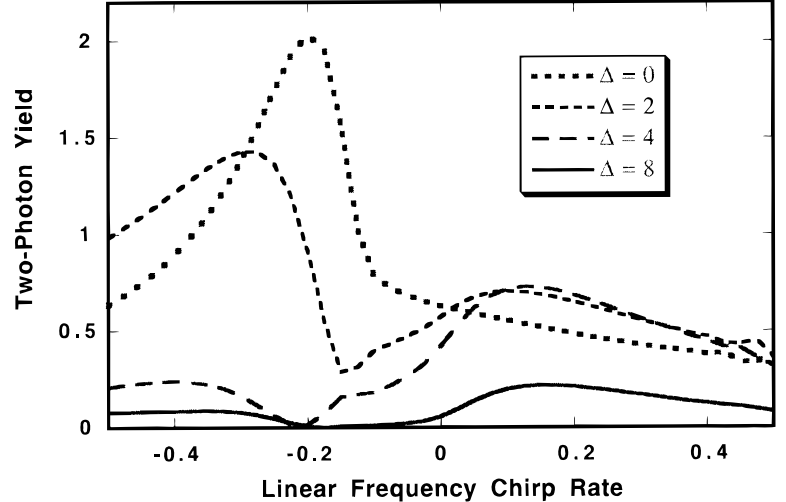

Figure 6. A plot of the two-photon yield evaluated from eqs 31 and 32 as a function of the linear frequency chirp rate $c^{\prime}$ for $\Gamma=5, \alpha=1$, and $P^{2} / \gamma=1$. The four curves corresponds to a resonant electric field $(\Delta=0)$, a near-resonant electric field $(\Delta=2)$, an off-resonant electric field $(\Delta=4)$, and a far-off-resonant electric field $(\Delta=8)$, respectively.

chirped for a large detuning $\Delta=8$. (The curve for $\Delta=8$ is shown with a magnification of 10 times.) As will be discussed in section $\mathrm{G}$, the above theoretical predications agree well with experimental observations.

G. Three-Photon Absorption. We propose here a fourelectronic-state three-photon model as a possible interpretation for the recent report of a three-photon experiment. ${ }^{13}$ In the specific three-photon process discussed here, the first photon creates a wave packet on a resonant excited state $|1\rangle$ from the ground state $|0\rangle$, then the second and third photons bring the wave packet to a final state $|2\rangle$ via an off-resonant intermediate state $\left|2^{\prime}\right\rangle$. We assume that the two-photon excitation from $|1\rangle$ to $|2\rangle$ has an off-resonance detuning which is substantially larger than the pulse bandwidth (i.e., $\Delta^{\prime} \gg \Gamma$ ). On the basis of the results in section $\mathrm{B}$, this two-photon transition can be described by an effective field

$$
E_{\text {eff }}(t)=C^{\prime}\left(\Delta^{\prime}\right) E^{2}(t)
$$

where $C^{\prime}\left(\Delta^{\prime}\right)$ is a constant for a fixed power spectrum and will be omitted hereafter. Then, the population on the final state can be written as

$$
N_{2}=\left|\int_{-\infty}^{\infty} \mathrm{d} t_{2} \int_{-\infty}^{t_{2}} \mathrm{~d} t_{1} \mathrm{e}^{\mathrm{i} \omega_{2}\left(x_{1}\right) t_{2}+\mathrm{i} \omega_{1}\left(x_{0}\right) t_{1}} E^{2}\left(t_{2}\right) E\left(t_{1}\right)\right|^{2}
$$

which is similar to eq 30 except that $E^{2}\left(t_{2}\right)$ is used instead of $E\left(t_{2}\right)$. Since $E\left(t_{2}\right)$ and $E\left(t_{1}\right)$ have different powers in eq 36 , the change of integral variables is defined as $s=t_{2}-t_{1}$ and $3 t=$ $2 t_{2}+t_{1}$. Following the same argument as in section $\mathrm{F}$, we complete the integration of $t$ and thus obtain

$$
N_{2}=\frac{P^{3}}{\Gamma \tau^{2}}|C(\Delta)|^{2}
$$

where the $s$ integral is explicitly expressed as

$$
C(\Delta)=\int_{0}^{\infty} \mathrm{d} s \exp \left[-\gamma^{2} \frac{s^{2}}{3}-\frac{\left(\Delta-\alpha s^{2}\right)^{2}}{6 \gamma^{2}}+\mathrm{i}\left(\Delta-\alpha s^{2}\right) \frac{s}{3}\right]
$$

with $\gamma^{2}=1 / \tau^{2}+\mathrm{i} c$. Except for a few minor differences, such as the $1 / \tau^{2}$ dependence of the prefactor in eq 37 and the coefficients in the exponent of eq 38 , the two expressions in eq 37 and 38 are exactly the same as eq 31 and 32 . Consequently, all the conclusions drawn in section $\mathrm{F}$ are applicable to the three- photon process formulated here. Since the two-photon excitation from electronic states $|1\rangle$ to $|2\rangle$ is treated effectively as a single-photon absorption, the underlying physics of the threephoton process discussed here is essentially the same as the coherent intrapulse pump-probe process discussed in section F. Thus, the observations in the three-photon experiment by Yakovlev, Bardeen, Che, Cao, and Wilson ${ }^{13}$ are consistent with the predictions of chirp dependence in this type of multiphoton intrapulse pump-probe process.

\section{Conclusion}

The single-photon stationary and nonstationary two-level models are generalized to three-level models to treat ultrafast two-photon processes including intrapulse pump-dump and intrapulse pump-probe. Within the theoretical framework, three different intrapulse coherence effects (sequential-resonance, time-delay, and chirp following) are identified and the subtle chirp dependence in a near-resonant intrapulse pumpprobe process is shown to be the combined consequence of these three dynamical mechanisms. The resulting theoretical predictions lead to a possible explanation for recent three-photon experimental results. ${ }^{13}$ More importantly, the theoretical approaches developed in this paper provide a general recipe for studying other ultrafast multiphoton processes. It should also be noted that the relatively high intensity used in multiphoton experiments may well extend into the strong-response regime such that the multiphoton perturbative analysis formulated in this paper has to be extended accordingly. In fact, interesting phenomena unexpected in the weak field regime, such as molecular $\pi$ pulses, can be observed in strong-field quantum mechanical calculations. ${ }^{12}$ Another possible direction to be explored is coherence effects in frequency-dispersed ultrafast nonlinear optical experiments. The rich physics revealed in multiphoton and strong-response femtosecond processes will be an exciting subject for future study.

Acknowledgment. We thank Dr. Bardeen and Dr. Yakovlev for helpful discussions.

\section{References and Notes}

(1) Zewail, A. H. In Femtosecond Chemistry; Manz, J., Wöste, L., Eds.; Springer-Verlag: Weinheim, 1995; p 15.

(2) Mukamel, S. The Principles of Nonlinear Optical Spectroscopy; Oxford University Press: New York, 1995.

(3) Bersohn, R.; Zewail, A. H. Ber. Bunsen-Ges. Phys. Chem. 1988, 92,373

(4) Fain, B.; Lin, S. H.; Hamer, N. J. Chem. Phys. 1989, 91, 4485.

(5) Pollard, W. T.; Lee, S.-Y.; Mathies, R. A.; J. Chem. Phys. 1990, 92, 4012.

(6) Lee, S. Y.; In Femtosecond Chemistry; Manz, J., Wöste, L., Eds.; Springer-Verlag: Weinheim, 1995; p 273.

(7) Kohler, B.; Yakovlev, V. V.; Che, J.; Krause, J. L.; Messina, M.; Wilson, K. R.; Schwentner, N.; Whitnell, R. M.; Yan, Y. J. Phys. Rev. Lett. 1995, 74, 3360.

(8) Sterling, M.; Zadoyan, R.; Apkarian, V. A. J. Chem. Phys. 1996, 104, 6497

(9) Cao, J.; Wilson, K. R. J. Chem. Phys. 1997, 106, 5062

(10) Ruhman, S.; Kosloff, R. J. Opt. Soc. Am. B 1990, 7, 1748.

(11) Cerullo, G.; Bardeen, C. J.; Wang, Q.; Shank, C. V. Chem. Phys. Lett. 1996, 262, 362.

(12) Cao, J.; Bardeen, C. J.; Wilson, K. R. Phys. Rev. Lett. 1997. In press.

(13) Yakovlev, V. V.; Bardeen, C. J.; Che, J.; Cao, J.; Wilson, K. R. J. Chem. Phys. 1997, submitted for publication.

(14) Cina, J. A.; Smith, T. J. J. Chem. Phys. 1993, 98, 9211.

(15) Smith, T. J.; Cina, J. A. J. Chem. Phys. 1996, 104, 1272

(16) Banin, U.; Bartana, A.; Ruhman, S.; Kosloff, R. J. Chem. Phys. 1994, 101, 8571 .

(17) Broers, B.; van Linden, H. B.; van den Heuvell, X.; Noordam, L. D. Opt. Commun. 1992, 91, 57.

(18) Brumer, P.; Shapiro, M. Chem. Phys. 1989, 139, 221. 